OPEN ACCESS

Edited by:

Wenhui Song,

China University of Petroleum

(Huadong), China

Reviewed by:

Meng Li,

China University of Petroleum

(Huadong), China

Tao Zhang,

Southwest Petroleum University,

China

*Correspondence:

Guanglong Sheng

shenggl2019@yangtzeu.edu.cn

Specialty section:

This article was submitted to

Economic Geology,

a section of the journal

Frontiers in Earth Science

Received: 29 July 2021

Accepted: 13 August 2021

Published: 07 October 2021

Citation:

Liu J, Lu M and Sheng G (2021) Description of Fracture Network of Hydraulic Fracturing Vertical Wells in

Unconventional Reservoirs.

Front. Earth Sci. 9:749181.

doi: 10.3389/feart.2021.749181

\section{Description of Fracture Network of Hydraulic Fracturing Vertical Wells in Unconventional Reservoirs}

\author{
Jinghua Liu ${ }^{1}$, Mingjing $L u^{2,3}$ and Guanglong Sheng ${ }^{1 *}$ \\ ${ }^{1}$ School of Petroleum Engineering, Yangtze University, Wuhan, China, ${ }^{2}$ Petroleum Engineering Technology Research Institute of \\ Shengli Oilfield, Dongying, China, ${ }^{3}$ Postdoctoral Scientific Research Working Station of Shengli Oilfield, Dongying, China
}

Based on the distribution of complex fractures after volume fracturing in unconventional reservoirs, the fractal theory is used to describe the distribution of volume fracture network in unconventional reservoirs. The method for calculating the fractal parameters of the fracture network is given. The box dimension method is used to analyze a fracturing core, and the fractal dimension is calculated. The fractal index of fracture network in fracturing vertical wells are also firstly calculated by introducing an analysis method. On this basis, the conventional dual-media model and the fractal dual-media model are compared, and the distribution of reservoir permeability and porosity are analyzed. The results show that the fractal porosity/permeability can be used to describe the reservoir physical properties more accurately. At the same time, the flow rate calculating by conventional dual-media model and the fractal dual-media model were calculated and compared. The comparative analysis found that the flow rate calculated by the conventional dual-media model was relatively high in the early stage, but the flow rate was not much different in the later stage. The research results provide certain guiding significance for the description of fracture network of volume fracturing vertical well in unconventional reservoirs.

Keywords: unconventional reservoirs, hydraulic fracturing, fracture network, fractal dimension, fractal index

\section{INTRODUCTION}

Unconventional reservoirs in the world are rich in resources, and have poorly reservoir porosity/ permeability (Song et al., 2018; Wang et al., 2019; Chen et al., 2020). It is necessary to adopt stimulation enhancement measures to improve the physical properties in order to achieve economic development (Li et al., 2015). Considering the distribution of natural fractures in unconventional reservoirs, through technologies such as optimized displacement and low liquid viscosity, the net pressure in the fracture during volume fracturing is achieved, and the fracture opening conditions are achieved (Zhang et al., 2021). So as to extend along the wall of the main fracture and communicate with multi-scale secondary fractures, then the complex fracture network is finally formed in the formation (Manchanda et al., 2020; Qi et al., 2020; Sheng et al., 2020).

Scholars have studied the distribution of fracture network of volume fracturing from many aspects. Cheng et al. used the discrete fracture network model and line network model to characterize the complex fracture network of volume fracturing in shale reservoirs, and obtained the fracture network geometry parameter (Cheng et al., 2013). Wang et al. used numerical simulation to study the factors affecting the productivity of volume fracturing vertical wells, and the results showed that the complex fracture network formed after volume fracturing in tight reservoirs can greatly increase the productivity (Wang et al., 2013). Li et al. used dual-media 
numerical simulation to simulate the fracture network from volume fracturing and optimize the parameters of fracture network (Li, 2020). They believed that the dual-media simulation method can accurately describe the complex fracture network composed of native natural fractures and artificial hydraulic fractures. The dual-media simulation method can also reflect the flow characteristics within fracture network and matrix after volume fracturing. Based on the characteristics of complex fracture network and flow mechanism of volume fracturing in tight reservoirs, Su et al. proposed a coupled dual-media composite flow model considering the limited stimulated reservoir volume, and optimized the parameters of fracture network (Su et al., 2014).

The previous research fully demonstrated the influence of the fracture network on reservoir flow and made a preliminary description of fracture network (Li, 2020). However, the distribution of fracture network cannot describe by a simple approach, such as analytical equations (Meng et al., 2020). The complex fracture network was formed based on communicating and extending natural fractures ( $\mathrm{Li}$ et al., 2020), so the fracture network structure is consistent with the distribution of natural fractures in the reservoir. Pfeiferper and Avnir (1983), Katz and Thompson (1985), Krohn (1988) used adsorption method, electron microscope observation and mercury intrusion method to study the microscopic pore and natural fracture structure of unconventional reservoirs. A large number of research results show that the microstructure of unconventional reservoirs has fractal characteristics (Song et al., 2019), which can be studied by fractal theory. Yortsos et al. used the fractal reservoirs for the first time to describe natural fractured reservoirs (Chang and Yortsos, 1990). Aprilian et al. (1993) and Acuna et al. (1995) respectively used fractal reservoir-based well testing analysis methods to explain the well test results of complex reservoirs that were difficult to explain by conventional methods, and obtained results consistent with the actual data. Wang et al. introduced the fractal theory into trilinear flow model, and proposed a mathematical model considering complex fractures and fractal flow for pressure transient analysis of fractured horizontal wells in unconventional reservoirs. In the work, the fractal dimension and fractal index were used directly to describe the fractal fracture network (Wang et al., 2015). To tell the truth, the calculation of fractal dimension and fractal index is more important for fracture network description. The fractal dimension is usually calculated by box counting method, which is based on the specific fracture geometry. Combined with the inversion method of fracture network, the fractal dimension can be easier calculated. However, the fractal index is hardly to obtain. The fractal index is usually obtained by random walker method (O Shaughnessy and Procaccia, 1985), which is not easier in the calculation of fractal index of fracture network. Sheng et al. further gave the calculation method of the heterogeneity fractal index based on the fractal dimension, and obtained the fracture porosity and permeability distribution with the fractal fracture morphology (Sheng et al., 2019). This work presents an effective method to determine the fractal index, and make the fractal theory more reasonable to apply to the

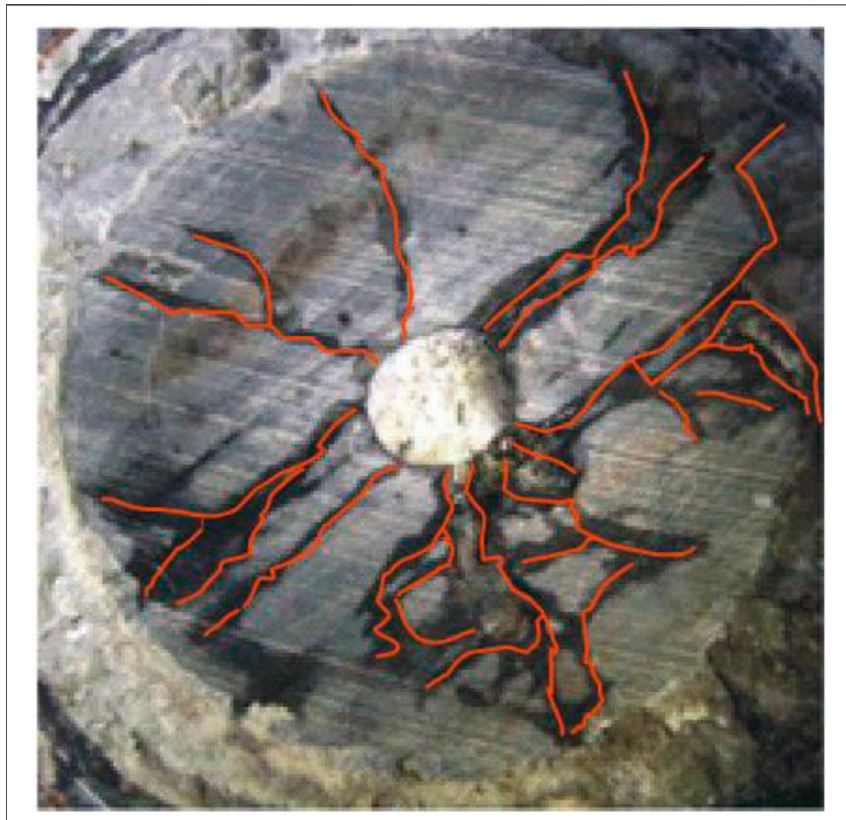

FIGURE 1 | Fracture network morphology in unconventional reservoirs (Guo et al., 2015).

description of fracture network. However, there is no work combine the fractal index calculation method with flow simulation.

Therefore, considering the fracture network morphology in unconventional reservoirs, this paper uses fractal theory to describe the distribution of induced fracture network in unconventional reservoirs. The calculation method of fractal parameters is given, and the distribution of physical properties and the production rate from conventional dual-media flow model and the fractal dualmedia flow model are compared and analyzed.

\section{DESCRIPTION OF FRACTURE NETWORK IN UNCONVENTIONAL RESERVOIRS}

Researches show that the microstructure of natural fractures in unconventional reservoirs has fractal characteristics. Based on the characteristics of natural fractures in unconventional reservoirs, Hydraulic fracturing communicate with multiple induced fractures and micro-fractures, and finally generates a complex fracture network in the reservoir. Therefore, the resulting fracture network presents a multi-scale distribution and shows a certain degree of self-similarity in a statistical sense (Figure 1). In the conventional model, the fracture density is used to characterize the number of induced fractures, but the fracture density cannot accurately describe the tortuosity and self-similarity of the fracture network. Fractal geometry breaks through the traditional integer dimension thinking and has superiority in describing extremely complex geometric forms. When using fractal theory to characterize the structural characteristics of the fracture network, two parameters, fractal dimension and fractal index, are generally used. 

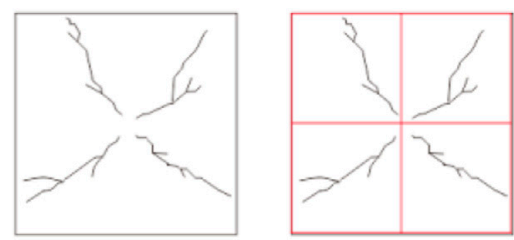

$R=L / 2$

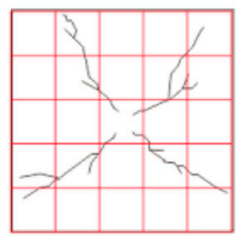

$R=L / 5$

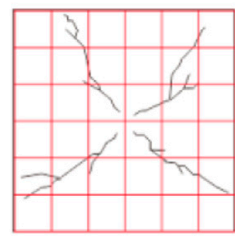

$R=L / 6$

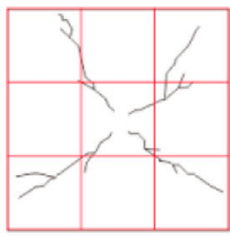

$R=L / 3$

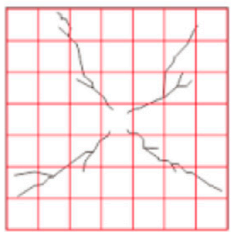

$R=L / 7$

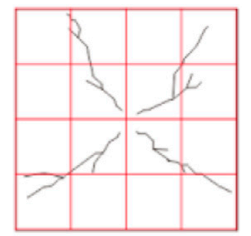

$R=L / 4$

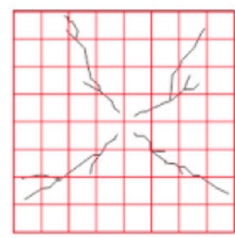

$R=L / 8$

FIGURE 2 | Fracture distribution in different squares.

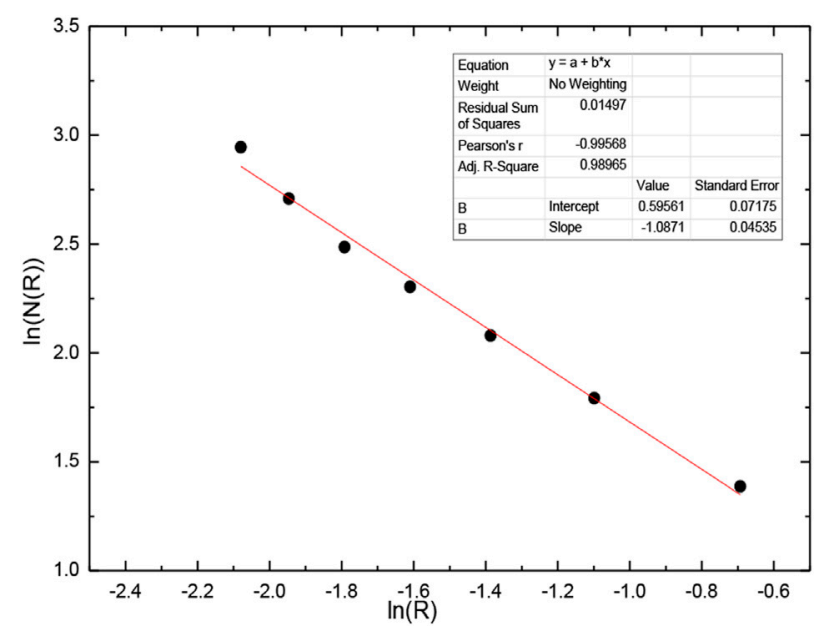

FIGURE 3 | The fractal dimension from box dimension method.

\section{Fractal Dimension}

The fractal dimension is often used in fractal geometry to reflect the density and complexity of fracture network. The experimental method to calculate the fractal dimension have the disadvantages of cumbersome operation when determining the fracture network parameters. The box dimension method based on the microseismic data is used to obtain the fractal dimension of the fracture network, which has a good effect. The specific process is: 1) divide the geometry into a grid with a square of length $R, 2$ ) count the number of grids with fractures in the grid $N(R), 3$ ) repeat the above process continuously by changing the size of $R$, and 4) the fractal dimension can be obtained by drawing the curve of $\ln (R)$ and $\ln (N(R))$

$$
d=-\frac{d(\ln (N(R)))}{d(\ln (R))}
$$

Where $R$ is the length of the square; $N(R)$ is the number of grids with fractures in the square of length $R$.
Assuming that the fractures are distributed inside a square with side length $L$ after hydraulic fracturing (the fractal dimension has nothing to do with the value of $L$, this work assumes $L=1$ ), as shown in Figure 2, the fracture distribution area is continuously divided.

Calculate the number of grids with fractures at different grid sizes $(R)$, and draw the curve of $\ln (R)$ and $\ln (N(R))$, as shown in Figure 3. It can be seen from the figure that the $\ln (R)$ and $\ln$ $(N(R))$ have a strong linear relationship, and the fractal dimension of the fracture network shown in Figure 3 is 1.086.

\section{Fractal Index}

Since there are many branch and bifurcations in fractal fractures, the longer the fluid flow in the fractures, the slower the flow rate will be. This characteristic in the fractal fracture network is called fractal anomalous diffusion. The fractal index is used to characterize the fractal anomalous diffusion. The value is usually estimated by random walking method, and its absolute value is between 0 and 1 . In reservoir simulation, the abnormal diffusion mainly affects the permeability.

Sheng et al. (2019) obtained the fractal index of fractal network distribution, which can be expressed as

$$
\theta=\ln \left(1+\frac{s_{f w}}{r_{w}}|2-d|\left(\frac{r}{r_{w}}\right)^{1-d}\right) / \ln \left(\frac{r}{r_{w}}\right)
$$

Where $r_{w}$ represents a reference length, $\mathrm{cm} ; r$ is the length from the reference point, $\mathrm{cm} ; d$ is the fractal dimension; $\theta$ is the fractal index; $s_{f w}$ is the fracture width at the reference point, $\mathrm{cm}$.

\section{Fractal Porosity/Permeability Equation}

Based on the fractal dimension and fractal index, the fractal permeability and porosity equation of the reservoir with complex fracture network can be obtained

$$
k_{f}(x)=k_{w}\left(\frac{r}{r_{w}}\right)^{-\frac{d(\ln (N(R)))}{d(\ln (R))}-\ln \left(1+\frac{s_{f w}}{r_{w}}|2-d|\left(\frac{r}{r_{w}}\right)^{1-d}\right) / \ln \left(\frac{r}{r_{w}}\right)-2}
$$




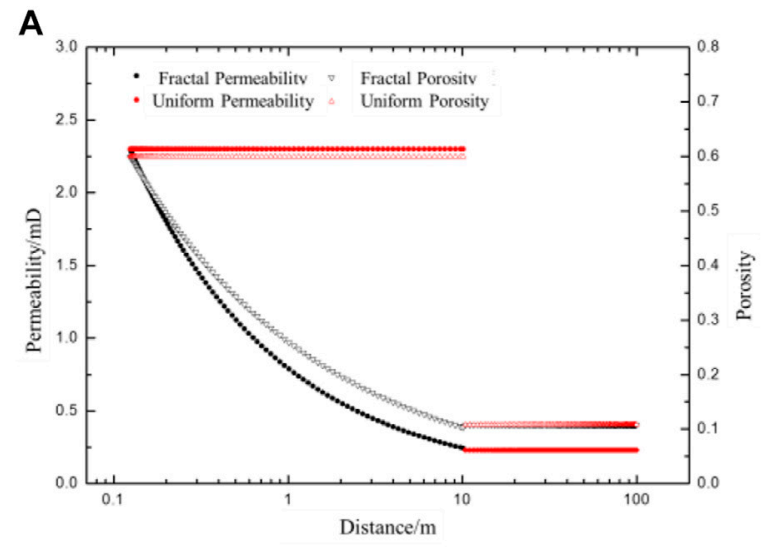

Physical properties distribution

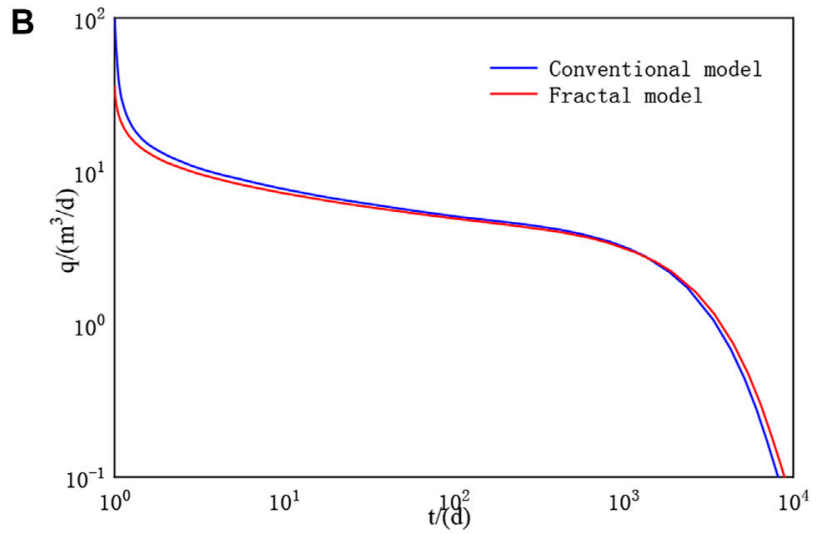

Production curve

FIGURE 4 | Physical properties distribution and production in stimulated unconventional reservoirs. (A) is the physical properties distribution, (B) is the production curve.

$$
\phi_{f}=\phi_{w}\left(\frac{r}{r_{w}}\right)^{-\frac{d(\ln (N(R)))}{d(\ln (R))}-2}
$$

Where $k_{f}$ is the equivalent permeability of fracture network in the dual media model, $\mathrm{mD} ; \phi_{f}$ is the equivalent porosity of fracture network in the dual media model.

\section{PHYSICAL PROPERTIES AND PRODUCTION OF RESERVOIRS WITH FRACTAL FRACTURE NETWORK Physical Properties Distribution}

The fractal theory is used to accurately describe the physical parameters of fracture network in unconventional reservoirs. Assuming that a well is in the center of a circular reservoir, the wellbore radius is $12.4 \mathrm{~cm}$, the permeability at the wellbore is $2.3 \mathrm{mD}$, the porosity at the wellbore is 0.6 , the radius of stimulated reservoir is $10 \mathrm{~m}$, the reservoir permeability is $0.23 \mathrm{mD}$, the reservoir porosity is 0.108 , and the reservoir radius is $1000 \mathrm{~m}$, the fractal dimension is 1.8 and fractal index is 0.1 . Plotting the distribution of porosity and permeability in the reservoir is shown in Figure 4A. When the conventional dual-media flow model is used to describe the reservoir physical properties, the reservoir permeability and porosity will undergo abrupt changes at the boundary of the stimulated area. While when the fractal theory is adopted to describe the physical properties of the stimulated area, the permeability and porosity of the reservoir can smoothly transition between the stimulated boundary and the unstimulated boundary, which is more consistent with the actual geological characteristics. Therefore, the dual-media flow model can more accurately characterize the fluid flow in the stimulated unconventional reservoirs.

\section{Dynamic Production of Reservoirs With Fractal Fracture Network}

Assuming that there is a vertical well in the middle of a circular reservoir, producing at constant pressure. The initial reservoir pressure is $20 \mathrm{MPa}$, and the bottom hole pressure is $8 \mathrm{MPa}$. Using the above parameters and the calculated fractal dimension, daily production rate from the conventional dual-media flow model and fractal dual-media flow model are calculated, as shown in Figure 4B. It can be seen from the figure that the difference between the production calculated by the two models occurs in the early stage. When considering the distribution of the fractal fracture network, the early stage of production is smaller than the calculation result of the conventional dual-media flow model. However, it is close to the actual production data of the stimulated unconventional reservoirs. The results calculated by the two models are relatively similar in the middle and late stages of production.

\section{CONCLUSION}

1) Based on the complex fracture distribution of unconventional reservoirs after hydraulic fracturing, this paper uses fractal theory to describe the distribution of fracture networks, and proposes a calculation method of fractal parameters for hydraulic fracturing vertical wells in unconventional reservoirs.

2) The fractal theory are used to describe the distribution of fracture network, it can be seen from the physical property distribution that using fractal theory to describe the physical properties of stimulated area can make the reservoir permeability and porosity smoothly transition between the stimulated boundary and unstimulated boundary, which is different from the conventional methods. 
3) The production changes from the conventional dual-media flow model and the fractal dual-media flow model were calculated respectively. The comparative analysis showed that the difference mainly occurred in the early stage of production, and the production considering the fractal fracture network is relatively small.

\section{DATA AVAILABILITY STATEMENT}

The original contributions presented in the study are included in the article/supplementary material, further inquiries can be directed to the corresponding author.

\section{REFERENCES}

Acuna, J. A., Ershaghi, I., and Yortsos, Y. C. (1995). Practical Application of Fractal Pressure Transient Analysis of Naturally Fractured Reservoirs. Spe Formation Eval. 10 (03), 173-179. doi:10.2118/24705-pa

Aprilian, S., Abdassah, D., and Mucharam, L. (1993). Application of fractal reservoir model for interference test analysis in Kamojang geothermal field (Indonesia). Houston, TX, USA: Publ by Society of Petroleum Engineers (SPE).

Chang, J., and Yortsos, Y. C. (1990). Pressure Transient Analysis of Fractal Reservoirs[J]. SPE Formation Eval. 5 (1), 31-38. doi:10.2118/18170-pa

Chen, Y., Zhao, H., Zhang, Q., Zhou, Y., Wang, H., Lei, S., et al. (2020). Development and Application of a Coupling Method for Well Pattern and Production Optimization in Unconventional Reservoirs. J. Circuit Syst. Comp. 29 (07), 2050105. doi:10.1142/s0218126620501054

Cheng, Y., Li, Y., and Shi, X. (2013). Analysis and application of fracture network models of volume fracturing in shale gas reservoirs[J]. Nat. Gas Industry 33 (9), 53-59.

Guo, T., Zhang, S., Ge, H., Wang, X., Lei, X., and Xiao, B. (2015). A new method for evaluation of fracture network formation capacity of rock. Fuel 140, 778-787. doi:10.1016/j.fuel.2014.10.017

Katz, A. J., and Thompson, A. H. (1985). Fractal Sandstone Pores: Implications for Conductivity and Pore Formation. Phys. Rev. Lett. 54 (12), 1325-1328. doi:10.1103/physrevlett.54.1325

Krohn, C. E. (1988). Fractal measurements of sandstones, shales, and carbonates. J. Geophys. Res. 93 (B4), 3297-3305. doi:10.1029/jb093ib04p03297

Li, Q., Xing, H., Liu, J., and Liu, X. (2015). A review on hydraulic fracturing of unconventional reservoir. Petroleum 1 (1), 8-15. doi:10.1016/ j.petlm.2015.03.008

Li, Y. (2020). Simulation of the interactions between multiple hydraulic fractures and natural fracture network based on Discrete Element Method numerical modeling. Energy Sci Eng 8 (8), 2922-2937. doi:10.1002/ese3.712

$\mathrm{Li}, \mathrm{Z}$., Li, X., Yu, J., and Cao, W. D. (2020). Influence of existing natural fractures and beddings on the formation of fracture network during hydraulic fracturing based on the extended finite element method[J]. Geomechanics Geophys. GeoEnergy Geo-Resources 6 (4), 58. doi:10.1007/s40948-020-00180-y

Manchanda, R., Shrivastava, K., and Zheng, S. (2020). A new mechanism for the formation of hydraulic fracture swarms. The Woodlands, TX, United states: Society of Petroleum Engineers.

Meng, M., Chen, Z., Liao, X., Wang, J., and Shi, L. (2020). A well-testing method for parameter evaluation of multiple fractured horizontal wells with non-uniform fractures in shale oil reservoirs. Adv. Geo-energy Res. 4 (2), 187-198. doi:10.26804/ager.2020.02.07

O Shaughnessy, B., and Procaccia, I. (1985). Diffusion on fractals[J]. Phys. Rev. a 32 (5), 3073.

Pfeiferper, P., and Avnir, D. (1983). Chemistry non-integral dimensions between two and Three[J][J]. J. Chem. Phys. 79 (7), 3369-3558. doi:10.1063/1.446210

Qi, J., Zhang, L., Zhang, K., Li, L., and Sun, J. (2020). The application of improved differential evolution algorithm in electromagnetic fracture monitoring. $A d v$. Geo-energy Res. 4 (3), 233-246. doi:10.46690/ager.2020.03.02

\section{AUTHOR CONTRIBUTIONS}

JL: Conceptualization, Methodology, Software, ML: Conceptualization, Methodology, Software, Investigation, Visualization, Project administration, Data Curation GS: Supervision, Funding acquisition.

\section{FUNDING}

This study was supported by the National Natural Science Foundation of China (52004033).

Sheng, G., Su, Y., and Wang, W. (2019). A new fractal approach for describing induced-fracture porosity/permeability/compressibility in stimulated unconventional reservoirs. J. Pet. Sci. Eng. 179, 855-866. doi:10.1016/ j.petrol.2019.04.104

Sheng, G., Su, Y., Zhao, H., and Liu, J. (2020). A unified apparent porosity/ permeability model of organic porous media: Coupling complex pore structure and multi-migration mechanism. Adv. Geo-energy Res. 4 (2), 115-125. doi:10.26804/ager.2020.02.01

Song, W., Wang, D., Yao, J., Li, Y., Sun, H., Yang, Y., et al. (2019). Multiscale imagebased fractal characteristic of shale pore structure with implication to accurate prediction of gas permeability. Fuel 241, 522-532. doi:10.1016/j.fuel.2018.12.062

Song, W., Yao, J., Ma, J., Sun, H., Li, Y., Yang, Y., et al. (2018). Numerical Simulation of Multiphase Flow in Nanoporous Organic Matter with Application to Coal and Gas Shale Systems. Water Resour. Res. 54 (2), 1077-1092. doi:10.1002/2017wr021500

Su, Y., Wang, W., and Sheng, G. (2014). Compound flow model of volume fractured horizontal Well[J]. Shiyou Xuebao/Acta Petrolei Sinica 35 (3), 504-510.

Wang, D., Yao, J., Chen, Z., Song, W., and Sun, H. (2019). Image-based core-scale real gas apparent permeability from pore-scale experimental data in shale reservoirs. Fuel 254, 115596. doi:10.1016/j.fuel.2019.06.004

Wang, W., Su, Y., and Mu, L. (2013). Influencing factors of stimulated reservoir volume of vertical wells in tight oil reservoirs[J]. Zhongguo Shiyou Daxue Xuebao (Ziran Kexue Ban)/Journal China Univ. Pet. (Edition Nat. Science) 37 (3), 93-97.

Wang, W., Su, Y., Sheng, G., Cossio, M., and Shang, Y. (2015). A mathematical model considering complex fractures and fractal flow for pressure transient analysis of fractured horizontal wells in unconventional reservoirs. J. Nat. Gas Sci. Eng. 23, 139-147. doi:10.1016/j.jngse.2014.12.011

Zhang, X., He, J., Deng, H., Fu, M., Xiang, Z., Peng, X., et al. (2021). Controls of interlayers on the development and distribution of natural fractures in lacustrine shale reservoirs: A case study of the Da'anzhai member in the Fuling area in the eastern Sichuan Basin[J]. J. Pet. Sci. Eng., 109224.

Conflict of Interest: Author ML is employed by SINOPEC.

The remaining authors declare that the research was conducted in the absence of any commercial or financial relationships that could be construed as a potential conflict of interest.

Publisher's Note: All claims expressed in this article are solely those of the authors and do not necessarily represent those of their affiliated organizations, or those of the publisher, the editors and the reviewers. Any product that may be evaluated in this article, or claim that may be made by its manufacturer, is not guaranteed or endorsed by the publisher.

Copyright $(2021 \mathrm{Liu}, \mathrm{Lu}$ and Sheng. This is an open-access article distributed under the terms of the Creative Commons Attribution License (CC BY). The use, distribution or reproduction in other forums is permitted, provided the original author(s) and the copyright owner(s) are credited and that the original publication in this journal is cited, in accordance with accepted academic practice. No use, distribution or reproduction is permitted which does not comply with these terms. 